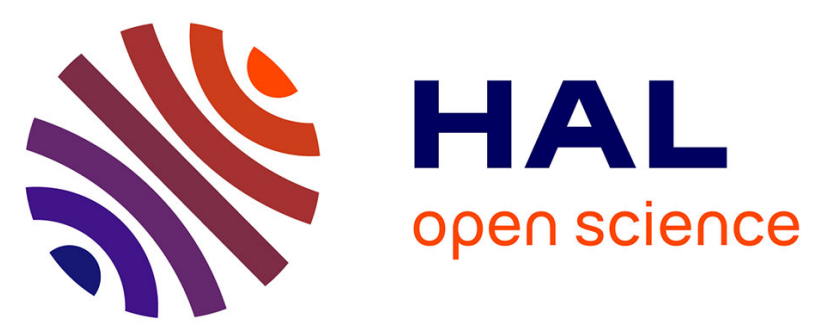

\title{
The dynamics of cortical folding waves and prematurity-related deviations revealed by spatial and spectral analysis of gyrification
}

Jessica Dubois, Julien Lefèvre, Hugo Angleys, François Leroy, Clara Fischer, Jessica Lebenberg, Ghislaine Dehaene-Lambertz, Cristina Borradori-Tolsa, François Lazeyras, Lucie Hertz-Pannier, et al.

\section{To cite this version:}

Jessica Dubois, Julien Lefèvre, Hugo Angleys, François Leroy, Clara Fischer, et al.. The dynamics of cortical folding waves and prematurity-related deviations revealed by spatial and spectral analysis of gyrification. NeuroImage, 2019, 185, pp.934-946. 10.1016/j.neuroimage.2018.03.005 . hal-01781242

\section{HAL Id: hal-01781242 \\ https://hal-amu.archives-ouvertes.fr/hal-01781242}

Submitted on 2 May 2018

HAL is a multi-disciplinary open access archive for the deposit and dissemination of scientific research documents, whether they are published or not. The documents may come from teaching and research institutions in France or abroad, or from public or private research centers.
L'archive ouverte pluridisciplinaire HAL, est destinée au dépôt et à la diffusion de documents scientifiques de niveau recherche, publiés ou non, émanant des établissements d'enseignement et de recherche français ou étrangers, des laboratoires publics ou privés. 


\section{Accepted Manuscript}

The dynamics of cortical folding waves and prematurity-related deviations revealed by spatial and spectral analysis of gyrification

Jessica Dubois, Julien Lefèvre, Hugo Angleys, François Leroy, Clara Fischer, Jessica Lebenberg, Ghislaine Dehaene-Lambertz, Cristina Borradori-Tolsa,

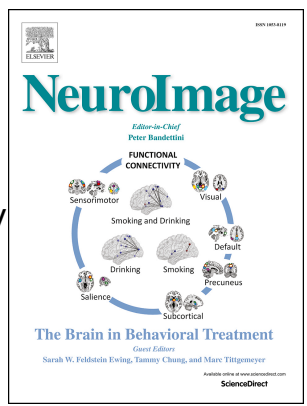
François Lazeyras, Lucie Hertz-Pannier, Jean-François Mangin, Petra Hüppi, David Germanaud

PII: S1053-8119(18)30190-3

DOI: 10.1016/j.neuroimage.2018.03.005

Reference: $\quad$ YNIMG 14774

To appear in: Neurolmage

Received Date: 5 September 2017

Revised Date: 14 February 2018

Accepted Date: 3 March 2018

Please cite this article as: Dubois, J., Lefèvre, J., Angleys, H., Leroy, Franç., Fischer, C., Lebenberg, J., Dehaene-Lambertz, G., Borradori-Tolsa, C., Lazeyras, Franç., Hertz-Pannier, L., Mangin, Jean.Franç., Hüppi, P., Germanaud, D., The dynamics of cortical folding waves and prematurity-related deviations revealed by spatial and spectral analysis of gyrification, Neurolmage (2018), doi: 10.1016/ j.neuroimage.2018.03.005.

This is a PDF file of an unedited manuscript that has been accepted for publication. As a service to our customers we are providing this early version of the manuscript. The manuscript will undergo copyediting, typesetting, and review of the resulting proof before it is published in its final form. Please note that during the production process errors may be discovered which could affect the content, and all legal disclaimers that apply to the journal pertain. 


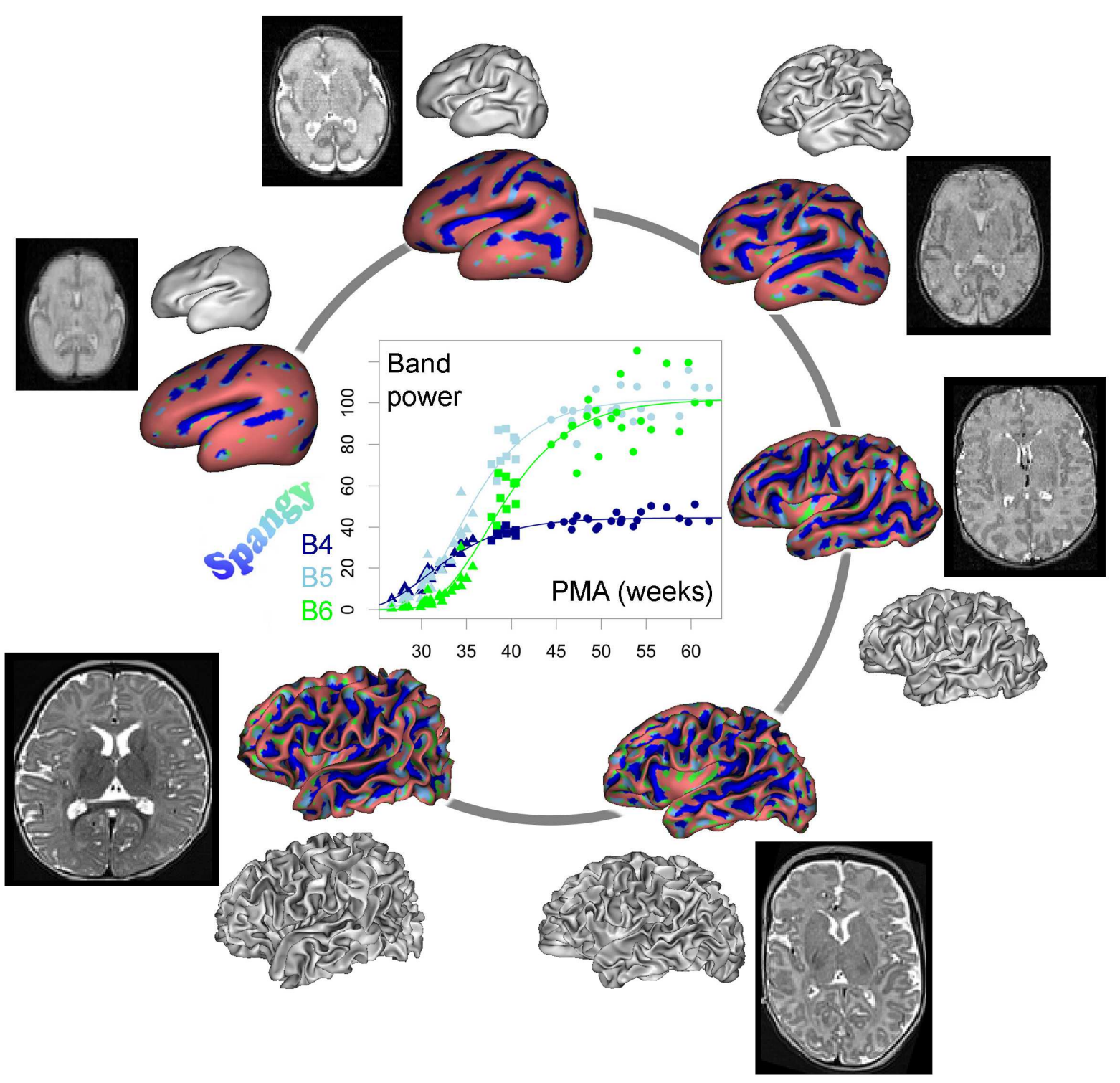




\title{
The dynamics of cortical folding waves and prematurity-related deviations revealed by spatial and spectral analysis of gyrification
}

\author{
Jessica Dubois $\mathrm{PhD}^{1}$, Julien Lefèvre $\mathrm{PhD}^{2}$, Hugo Angleys MSc ${ }^{1}$, François Leroy $\mathrm{PhD}{ }^{1}$, \\ Clara Fischer $\mathrm{MSc}^{3}$, Jessica Lebenberg $\mathrm{PhD}^{1,3}$, Ghislaine Dehaene-Lambertz MD-PhD ${ }^{1}$, \\ Cristina Borradori-Tolsa MD ${ }^{4}$, François Lazeyras $\mathrm{PhD}^{5}$, Lucie Hertz-Pannier MD-PhD ${ }^{6}$, \\ Jean-François Mangin $\mathrm{PhD}^{3}$, Petra Hüppi MD-PhD ${ }^{4}$, David Germanaud MD-PhD ${ }^{6-8}$
}

\section{Affiliations:}

1: INSERM, UMR992; CEA, NeuroSpin Center; University Paris Saclay, Gif-sur-Yvette, France

2: Institut de Neurosciences de la Timone, CNRS UMR7289, Aix-Marseille University, Marseille, France

3: CEA, NeuroSpin Center, UNATI; University Paris Saclay, Gif-sur-Yvette, France

4: Geneva University Hospitals, Department of Pediatrics, Switzerland

5: Geneva University Hospitals, CIBM, Switzerland

6: CEA, NeuroSpin, UNIACT, Neuropediatry team, Gif-sur-Yvette, France

7: INSERM, Sorbonne Paris Cité University (USPC), CEA, UMR 1129, Paris, France

8: Paris Diderot University (USPC), AP-HP, Robert-Debré Hospital, DHU Protect,

Department of pediatric neurology and metabolic diseases, Paris, France

\# of tables: 4

\# of figures: 5

\# of words in abstract: 198

\# of pages (including abstract, text, figures, tables, references): 27

Corresponding address:

Jessica Dubois, $\mathrm{PhD}$

CEA/SAC/DSV/I2BM/NeuroSpin/Cognitive Neuroimaging Unit U992

Bât 145, point courrier 156

91191 Gif-sur-Yvette, France

Email: jessica.dubois@centraliens.net

Phone: +33674574168

Fax: +33169087973

Running title: Waves of cortical folding

Article for the journal Neuroimage

Special issue Imaging Baby Brain Development

February $14^{\text {th }}, 2018$ 


\begin{abstract}
In the human brain, the appearance of cortical sulci is a complex process that takes place mostly during the second half of pregnancy, with a relatively stable temporal sequence across individuals. Since deviant gyrification patterns have been observed in many neurodevelopmental disorders, mapping cortical development in vivo from the early stages on is an essential step to uncover new markers for diagnosis or prognosis. Recently this has been made possible by MRI combined with post-processing tools, but the reported results are still fragmented. Here we aimed to characterize the typical folding progression ex utero from the pre- to the post-term period, by considering 58 healthy preterm and full-term newborns and infants imaged between 27 and 62 weeks of post-menstrual age. Using a method of spectral analysis of gyrification (SPANGY), we detailed the spatial-frequency structure of cortical patterns in a quantitative way. The modeling of developmental trajectories revealed three successive waves that might correspond to primary, secondary and tertiary folding. Some deviations were further detected in 10 premature infants without apparent neurological impairment and imaged at term equivalent age, suggesting that our approach is sensitive enough to highlight the subtle impact of preterm birth and extra-uterine life on folding.
\end{abstract}

\title{
Keywords
}

Cortex, development, sulcation, magnetic resonance imaging (MRI), prematurity

\section{Highlights}

- The progression of cortical folding was studied from pre- to post-term period.

- SPANGY provided a quantitative spectral and spatial analysis of cortical patterns.

- We showed three successive folding waves with different characteristic age points.

- This approach suggested deviations in primary folding in premature infants at TEA. 


\section{Introduction}

In the human brain, cortical folding is a fundamental and complex process that takes place mostly during the second half of pregnancy. So far, several hypotheses have been proposed on the factors that might trigger the formation of sulci (Dubois and DehaeneLambertz 2015; Fernandez, et al. 2016; Welker 1990; Zilles, et al. 2013). Cortical crowns, sulcal walls and fundi are already different in terms of structure and connectivity during development, with differences in neuronal orientation, axonal proliferative events, synaptogenesis, glial growth and cortical laminar differentiation, leading to higher volume of neuropil and thicker cortices in gyral crowns than in sulcal walls and fundi (Welker 1990). But whether these microstructural properties and changes are the cause or consequence of the folding process is still not understood. Mechanical models have also suggested plausible hypotheses on the folding driving force, such as a differential expansion of the outer cortical surface versus the inner core (Budday, et al. 2014; Tallinen, et al. 2014; Xu, et al. 2010), a differential growth of cortical sulci versus gyri (Lefèvre and Mangin 2010) or a tension applied radially by glial and axonal fibers to the cortical surface while it grows (Van Essen 1997).

Despite inter-individual variability of patterns across human individuals, the appearance of sulci seems to follow a relatively stable temporal sequence. Three successive folding "waves" have been described throughout development in fetuses and newborns (Chi, et al. 1977; Feess-Higgins and Laroche 1987), with the sequential appearance of early primary folds from 20 weeks of post-menstrual age (20w PMA), secondary folds from 32w PMA, and tertiary folds from term age (after 38w PMA). Since several genetic, epi-genetic and environmental morphogenetic factors likely influence this process, deviant gyrification patterns are observed in many neurodevelopmental disorders, and have been seen as potential neuroanatomical proxies of atypical development (Mangin, et al. 2010). Mapping cortical folding from the early stages on is thus an essential step to better understand these disorders and uncover new markers for diagnosis or prognosis.

Studying the developing human brain in vivo has recently become possible with the advent of non-invasive magnetic resonance imaging (MRI) combined with post-processing tools that extract cortical surfaces (Dubois and Dehaene-Lambertz 2015). The folding progression has been described in fetuses (Clouchoux, et al. 2012; Habas, et al. 2012; Rajagopalan, et al. 2011; Wright, et al. 2014), revealing intense and non-linear changes during 
the third gestational trimester, with differences across brain regions. Although normal fetuses ought to be the obvious ideal model to study typical brain development, in utero MRI is unfortunately still challenging in many respects (Girard and Chaumoitre 2012). Furthermore, it might not be straightforward to compare folding properties obtained in utero during the preterm period and ex utero during the post-term period. In fact, birth per se induces dramatic changes in physiological conditions, which might lead to different cortical configurations in pre-natal and post-natal brains as suggested by a recent study of fetuses and preterm newborns at birth (Lefèvre, et al. 2016). Given this obstacle to directly compare folding patterns before and after birth, early ex utero imaging of preterm newborns seems to be the only straightforward strategy to allow a continuous description of folding progression before and after term age. Studies on premature newborns have confirmed the dramatic folding increase during the pre-term period (Dubois, et al. 2008b; Kapellou, et al. 2006; Kersbergen, et al. 2016; Kim, et al. 2016a; Makropoulos, et al. 2015; Rodriguez-Carranza, et al. 2008). Nevertheless, most studies have considered healthy preterm newborns up to the term age, so several weeks after birth, making the comparison with typical development all the more disputable given obvious differences between in versus ex utero life. In full-term newborns, most tertiary sulci are still shallow compared with adults even if the global folding patterns look rather alike (Hill, et al. 2010). During early infancy, association cortices get further convoluted, with a slowdown between the first and the second year ( $\mathrm{Li}$, et al. 2014b). In brief, the reported developmental trajectory of gyrification is still fragmented, which justifies a continuous and extended description of folding changes from the pre-term to the post-term period, using similar processing and analysis methods across ages.

Technically speaking, several parameters have been introduced so far to characterize normal and pathological folding. Surface area, sulcal depth and a variety of surface ratios known as gyrification indices, provide direct measurements of cortical expansion and folding intensity, but give little information on shape. Recent search for more relevant measures has been discussed in fetuses for local curvature derivatives (Lefèvre, et al. 2016; Wright, et al. 2014), or in premature infants with or without brain injury (Shimony, et al. 2015). A major difficulty is that their sensitivity to brain volume or surface is intrinsically different (Rodriguez-Carranza, et al. 2008), questioning the way these size measures have to be taken into account for proper analysis and comparisons between groups. Furthermore, few quantitative tools have been proposed to disentangle between the different folding waves at the individual brain level. Supposedly corresponding to the location where sulci first fold, pits 
have been mapped in infants, with stable spatial distributions from term birth to 2 years of age despite increasing sulci depth (Meng, et al. 2014). Some attempts to characterize the emergence of secondary and tertiary sulci have been reported recently on the basis of highfrequency spatial features (Orasanu, et al. 2016) or shape complexity index (Kim, et al. 2016b). In adults, an original method of spectral analysis of gyrification (SPANGY) has been proposed to quantify the spatial-frequency structure of cortical folding (Germanaud, et al. 2012). Decomposing the curvature pattern, this approach has provided an anatomically relevant segmentation of folds based on the local spectral composition: while the lowfrequency components could be related to the global brain shape (Germanaud, et al. 2012; Lefèvre and Auzias 2015; Lefèvre, et al. 2014), high-frequency components were shown to account for folds shaping, successively matching primary and later developing folds (Germanaud, et al. 2012). Nevertheless, we still need to clarify whether this methodology accurately describes the folding waves at stages when sulci appear and deepen.

Aside from typical development, prematurity can be a cause of neurodevelopmental disabilities depending on several genetic and environmental compounding or protective factors. So far, the underlying alterations in brain development remain only partly understood (Ment, et al. 2009). Reductions in global cortical volume, surface area and folding measures have been reported in premature infants at term equivalent age (TEA) compared with fullterm newborns, and associated with lower gestational age at birth (Engelhardt, et al. 2015; Kim, et al. 2016a; Makropoulos, et al. 2015; Melbourne, et al. 2014; Padilla, et al. 2015). Decreases in gyrification index and global mean curvature have also been related with increasing abnormality scores at MRI in infants with varied outcome (Moeskops, et al. 2015). Nevertheless none had ruled out whether such folding deviations simply reflect the impaired overall brain size as studies of adult polymorphism might suggest (Germanaud, et al. 2012; Toro, et al. 2008). Disentangling direct from indirect size-related effects thus requires to match infants for both age and brain volume, considering a wide developmental period.

To investigate all these issues, this study first aimed to detail the folding progression from the pre-term period to early infancy (27w to $62 \mathrm{w}$ PMA), in 58 healthy newborns and infants, either born preterm and imaged at birth, or born full-term and imaged from birth on. As a "typical" model of ex utero development during the pre-term period, we considered premature neonates at birth (unaffected pregnancy, appropriate for gestational age and subsequently healthy) rather than fetuses, because the pre-natal and post-natal folding configurations are not comparable even at similar ages (Lefèvre, et al. 2016) while we aimed 
to provide a continuous description of folding during the pre-term and post-term periods. For that purpose, we examined the potential of SPANGY to reveal the successive waves of folding in a quantitative way. Our last goal was to evaluate whether this approach is sensitive enough to detect subtle folding deviations in a group of 10 preterms imaged at TEA, with reference to the developmental trajectories fitted in the typically growing babies.

\section{Material and methods}

\section{Subjects}

We studied four different populations of infants (Figure 1a), all from single pregnancies and imaged ex utero. The "pre-term group" consisted of 26 pre-term newborns (11 girls) without acute neurological complications nor brain lesions detected on MRI, and imaged within the first two weeks after birth, between 27w and 36w PMA (PMA being defined as the sum of gestational age GA at birth and post-natal age). As in our previous study (Lefèvre, et al. 2016), we considered that these newborns had "typical" patterns of cortical folding given the imaging circumstances (ex utero MRI during the pre-term period). The "term group" consisted of 9 full-term healthy newborns (4 girls), imaged within the first two days after birth at PMA between 38w and 40w. The "post-term group" consisted of 23 fullterm healthy infants ( 9 girls), imaged within the first post-natal weeks at PMA between $44 \mathrm{w}$ and $62 \mathrm{w}$. We assumed that typical developmental trajectories could be approached on the basis of these three cohorts.

In a second step, we assessed the effects of extra-uterine growth following pre-term birth, by considering 10 infants born prematurely (4 girls, GA at birth between $25 \mathrm{w}$ and $31 \mathrm{w}$ ) and imaged around TEA, at PMA between 38w and 41w ("TEA group"). No infant showed any lesion or ongoing acute injury on MRI at birth and at TEA, as assessed by PSH and a senior pediatric radiologist based on conventional high-resolution T1 and T2 weighted MR images, and DWI-derived maps of averaged diffusion coefficient. This TEA group was similar to each other typical group in regards to different age characteristic (Figure 1a). It was close to the pre-term group in terms of GA at birth, to the term group in terms of PMA at MRI, and to the post-term group in terms of post-natal age at MRI.

All MRI protocols detailed below were approved by the regional or institutional ethical committee, and all parents gave written informed consents. Newborns and infants were spontaneously asleep during MR imaging. Particular precautions were taken to minimize noise exposure by covering the ears with special "mini-muffs" or customized headphones. 


\section{Figure 1: Evolution of whole-brain morphometric parameters in the typical groups}

For the pre-term, term, post-term and TEA groups, post-menstrual age (PMA) at MRI is summarized according to gestational age (GA) at birth (a). The dots code for the group belonging (triangles=pre-term, squares=term, circles=post-term, stars=TEA), and the sex is indicated by the color (pink=girl, blue=boy). For the first three typical groups, hemispheric volume (b), cortical surface area (c), sulcation index (d) and SPANGY analyzed folding power (e) are presented as a function of PMA at MRI (the Gompertz models are outlined by the black curves in $\mathrm{d}$ and e). The distribution of power analyzed with SPANGY across the bands B0 to B6, i.e. the power spectrum, is presented in (f).
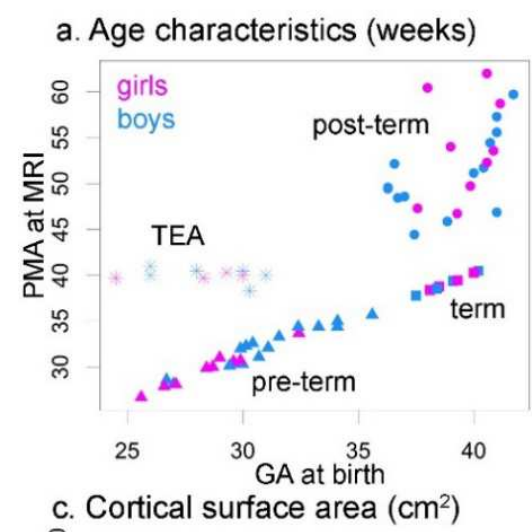

b. Hemispheric volume $(\mathrm{mL})$
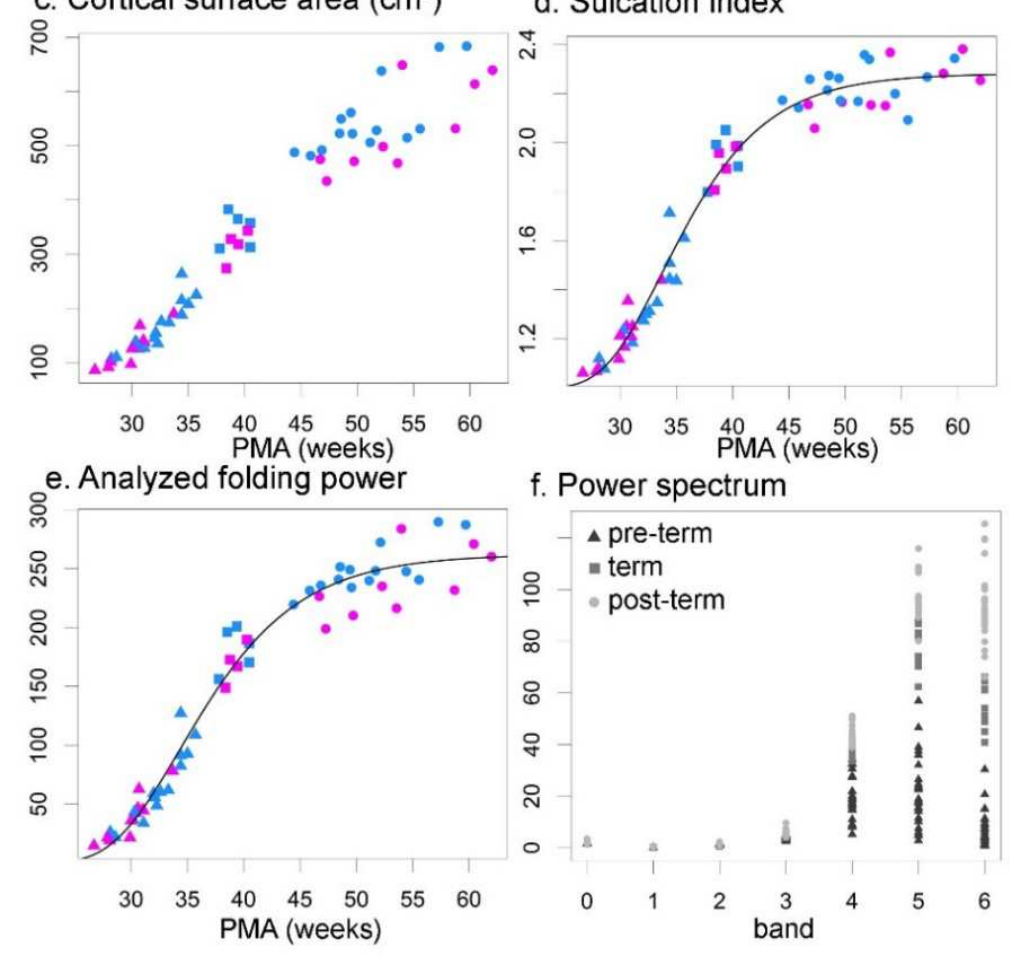

\section{f. Power spectrum}

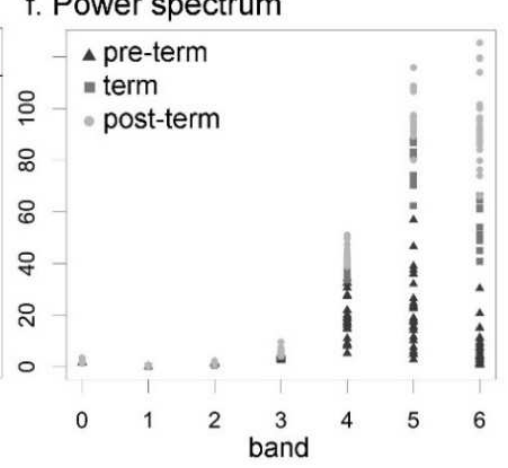

\section{Data acquisition}

For all subjects, T2-weighted (T2w) MR images were acquired with a high spatial resolution using a fast or a turbo spin echo sequence, in an interleaved way with slices concatenations. In the immature brain up to 6 months post-term, T2 weighting actually 
provides the best contrast between grey and white matter, in a more reliable way than T1 weighting (Dubois, et al. 2014) (Figure 2a).

For the pre-term group, acquisitions were performed in Geneva University Hospitals on a 1.5T-MRI system, either with the Eclipse, Intera or Achieva systems (Philips Medical Systems) and a receive quadrature head coil, or with the Avanto system (Siemens Medical System) and a 2-channel phased-array knee coil (see all the details in (Dubois, et al. 2010)). Note that the acquisition phase extended over a long period in view of the difficulty in imaging preterm newborns without neurological complications in the first days after birth, but that the newborns' age was homogeneously distributed across the different MRI systems. Coronal slices covering the whole brain were acquired with a spatial resolution of $0.7 \times 0.7 \times 1.5 \mathrm{~mm}$ or $0.8 \times 0.8 \times 1.2 \mathrm{~mm}$ (Dubois, et al. 2010).

Infants of the term and TEA groups were also imaged in Geneva University Hospitals, but on a 3T-MRI system (Tim Trio, Siemens Healthcare) with a 32-channel head coil. Coronal slices were acquired with a spatial resolution of $0.8 \times 0.8 \times 1.2 \mathrm{~mm}$ (TE/TR=150/4600ms).

Acquisitions in the post-term group were performed at NeuroSpin (CEA), on a 3TMRI system (Tim Trio, Siemens Healthcare) with a 32-channel head coil. Axial slices were imaged with a spatial resolution of $1 \times 1 \times 1.1 \mathrm{~mm}(\mathrm{TE} / \mathrm{TR}=149 / 4500 \mathrm{~ms})$ as detailed in (Kabdebon, et al. 2014).

\section{Data processing}

For analyses, we selected T2w images relatively free from motion artifacts (i.e. with only reduced artifacts in less than 1 slice over 4 or 5 depending on brain size), and these images were not corrected for these residual motion artefacts. All images were post-processed using tools implemented in BrainVISA software (Fischer, et al. 2012; Mangin, et al. 2004). The interface between cortex and white matter was segmented using semi-automatic procedures dedicated to pre-term newborns (Dubois, et al. 2008b), and infants beyond termequivalent age (Leroy, et al. 2011) (Figure 2b). Interactive manual corrections were carefully reviewed by a single operator (JD) in the places of low grey/white matter contrast or where the automatic algorithm made errors because of small inconsistencies across slices resulting from small motion artefacts. 3D meshes of left and right inner cortical surfaces were then provided for all newborns of the pre-term, term, post-term and TEA groups (Figure 2c). We then computed locally the mean cortical curvature as implemented in BrainVISA (Fischer, et al. 2012). 


\section{ACCEPTED MANUSCRIPT}

Figure 2: From MR images to SPANGY analyses

The successive post-processing steps are outlined for typical babies at different post-menstrual ages $(3$ pre-term newborns at 27w, 30w and 34w, 1 term newborn at 39w, 3 infants at 47w, 52w and $60 \mathrm{w}$, all girls). T2w images (a), segmentations of the boundary between cortex and white matter (b) and $3 \mathrm{D}$ reconstructions of the inner cortical surface (c) are presented with real proportions across newborns. SPANGY segmentations (d) are projected on smoothed cortical meshes (zoomed proportions), showing B4 sulci elements (deep blue) even in the youngest newborns. Zooms on the region around the central sulcus (e) highlight the progressive appearance of parcels for B5 (light blue) and B6 (green).

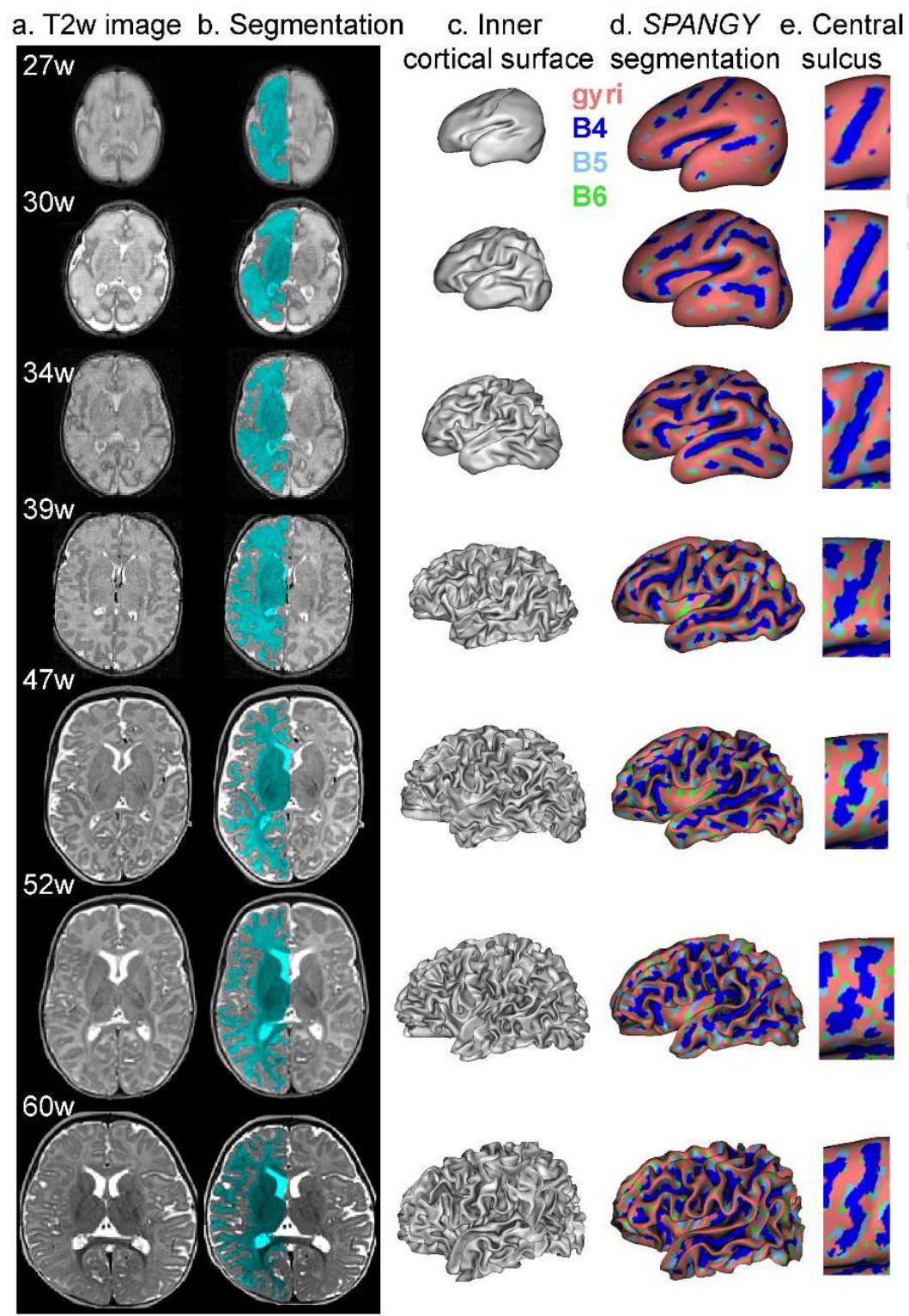

\section{SPANGY application in the developing brain}

Spectral analysis of gyrification (SPANGY) has been recently introduced

(Germanaud, et al. 2012) to analyze the cortical folding pattern in terms of spatial frequency 
composition, very much like a Fourier analysis would do for the voice oscillating signal in terms of temporal frequency composition. Precisely, SPANGY relies on the spectral decomposition of the cortical surface curvature on a basis of elementary patterns of increasing spatial frequencies, directly derived at the individual level for each hemispheric cortical mesh. In the adult brain, the curvature pattern is accurately characterized by a power spectrum of 7 bands of increasing frequencies (B0-B6), with successive frequency bands defined to fit a folding model in which new sulci coarsely appear in between two existing ones, hence doubling the pattern frequency. The low frequency bands (B0-B3) are related to the global brain shape, and the last 3 bands (B4-6) account for the folds shaping. The sulci can be further segmented by successive band filtering to identify the locally determinant frequency band: each vertex are labelled with the number of the band that determines whether it belongs to the sulcal or the gyral pattern (negative or positive curvature) based on a cumulative synthesis approach of the cortical folding pattern (Germanaud, et al. 2012). This defines a cortical segmentation in exclusive regions. In the adult brain, segments associated with B4, B5 and B6 appear to match essentially primary, secondary and tertiary folds respectively. For each subject, the fundamental wavelength corresponds to the whole hemisphere elongation (Lefèvre, et al. 2012), and the band-associated wavelengths are computed from it (on average in adults: 56mm, 28mm and 14mm for B4, B5 and B6 respectively) (Germanaud, et al. 2012). The SPANGY approach thus ensures proper inter-individual comparability of the spectral scale and of sulci elements for each band, whatever the brain size.

Here we performed SPANGY analysis in each hemisphere of all infants, in a similar way as in adults, with 7 bands being necessary and sufficient to describe the folding patterns as detailed in the results section. In fact we expected this number to be 7 or less since the folding patterns in the developing brain are seemingly less complex than in adults. On the reverse, we observed that in infants who already had quite complex folding (term and postterm groups), 7 bands were required not to miss relevant folding information.

\section{Data analyses}

\section{i. Whole-brain morphological and SPANGY parameters}

To characterize the global growth in each infant, we first measured a proxy of brain size: the hemispheric volume defined as the volume inside the closed outer cortical surface (at the interface with the cerebro-spinal fluid) (Fischer, et al. 2012; Germanaud, et al. 2012). We 
also computed the area of the inner cortical surface and a sulcation index (SI) defined as the ratio between the inner area and the surface area after morphological closing.

As for SPANGY in the spectral domain, we computed the analyzed folding power (AFP: sum of powers associated with the 7 bands B0-B6) and the spectral powers associated with B4, B5 and B6 bands (Germanaud, et al. 2012). We evaluated the proportions between those band powers through ratios referenced to the analyzed power. In the spatial domain (i.e. on the meshes), we described the localization of sulci elements (parcels) associated with B4, B5 and B6 bands, in comparison with the expected locations of primary, secondary and tertiary folds throughout development. We reported the B4-B6 corresponding wavelengths, which characterize the fold sizes and are supposed to directly rely on brain size. We also computed the B4-B6 numbers of parcels over the whole hemisphere, which inform about the fold quantity and are a direct marker of the gyrification progression.

Because only a few small-scale inter-hemispheric asymmetries were detected (Supplementary Information \#1), left and right parameters were averaged in all analyses.

\section{ii. Developmental patterns}

To outline typical patterns of brain growth over the pre-term to post-term period, we assessed age-related changes in both spatial and spectral parameters in a cross-sectional design considering the 58 infants of the 3 first groups. For each parameter, we performed analyses of co-variance (ANCOVA) with age as co-variable and sex as co-factor to assess differences between girls and boys. Since trajectories for some parameters appeared strongly non-linear (growth being the slowest at the youngest and oldest ages), we also modelled these changes with Gompertz functions as proposed for the growth of organs (Luecke, et al. 1995;

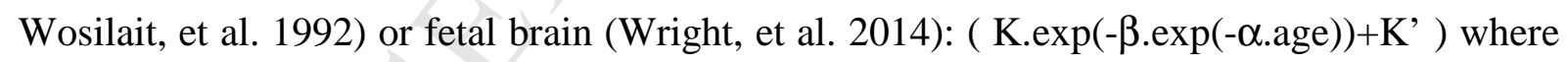
$\alpha, \beta, \mathrm{K}$ and $\mathrm{K}^{\prime}$ are constants to be fitted. Gompertz models were preferred to logistic functions because age-related evolutions were hardly symmetric. For each parameter, three age points of interest were computed from this modeling: the acceleration point, the inflexion point and the asymptotic deceleration point (Mischan, et al. 2011). The inflexion point corresponds to the vanishing point of the second derivative: $(\log (\beta) / \alpha)$. Acceleration (resp. deceleration) point corresponds to the first (resp. the last) maximum of the third derivative, and can be computed as: $(\log (\beta / \mathrm{r}) / \alpha)$ where $\mathrm{r}$ is the last (resp. the first) root of the polynomial: ( $\left.\mathrm{X}^{3}-6 \mathrm{X}^{2}+7 \mathrm{X}-1\right)(\mathrm{r} \approx 4.491$ and 0.166 respectively). Confidence intervals of these three points were obtained by Monte-Carlo simulations (Lambert, et al. 2012). 
Further approaches were considered to identify developmental sub-periods showing specific features of folding or discontinuities. Proportion of each band power was modelled as the ratio between the Gompertz functions for band power and for AFP, and we identified relevant age points based on the curves observation. We also investigated whether distinct sub-periods could be identified by integrating the complementary information provided by the three spectral bands. All 58 babies were clustered based on B4-6 power proportions with a kmeans algorithm and without any information on the babies' age. We expected a priori 4 clusters throughout the 27-62w period: 1 cluster corresponding mainly to primary folding, 2 clusters corresponding to the appearance of secondary and tertiary folds respectively, and 1 cluster corresponding to the folding slowdown. We compared the ages delimited by the 4 provided clusters with previous descriptions of the folding process (Chi, et al. 1977). For each cluster, we also investigated the relationship between cortical surface area $(S)$ and hemispheric volume $(V)$, since the inter-individual variation in cortical folding can be analyzed in terms of scaling (shape as a function of size) rather than growth (shape as a function of age). To demonstrate the non-proportional scaling called allometry (Awate, et al. 2009; Germanaud, et al. 2012; Kapellou, et al. 2006; Lefèvre, et al. 2016; Paul, et al. 2014; Toro, et al. 2008), here we used a power law modeling ( $S=b V^{a}$ ), and the scaling exponent (a) was measured through logarithmic linearization and compared with the one under the hypothesis of strictly proportional geometric dependence between surface and volume $(a=2 / 3)$.

\section{iii. Atypical folding in premature infants at term equivalent age}

For each morphometric and SPANGY parameter, we compared the 10 preterms at TEA with the 58 typical babies. To better take into account the inter-individual variability resulting from cross-sectional data for these latter subjects, we performed the comparison based on the developmental trajectories defined with Gompertz modeling. As detailed in (Germanaud, et al. 2014), we used a shuffle-and-split (S\&S) algorithm since this iterative resampling-based non-parametric test allows comparisons involving a model regression for small group size and important size difference between groups (here a 1:6 ratio). Because the numerical combination of two models introduced an artificial variance preventing us to compute reliable p-values with the S\&S algorithm, power proportions were compared with Ttests limited to this age window as detailed in Supplementary Information \#2. Effect sizes were then characterized based on Cohen's d. 


\section{Results}

\section{Whole-brain morphometry analyses in typical babies}

Over the developmental period from $27 \mathrm{w}$ to $62 \mathrm{w}$ PMA, whole hemispheric volume and cortical surface area increased significantly, with lower values in girls than in boys particularly in the term and post-term groups (Figure 1b,c; Table 1). Parameters showed high continuity across the groups although we analyzed cross-sectional data and despite differences in image acquisition and post-processing.

Measures of folding (SI and AFP) also increased with age, but showed more equivocal differences between girls and boys (Figure 1d,e; Table 1). Modeling with Gompertz functions highlighted similar characteristic age points, with an acceleration of folding around 25-26w, an inflection around 34-35w, and a deceleration around 43-46w (Table 2), suggesting an intense folding progression during the third gestational trimester slowing-down during early infancy. Nevertheless, with these global folding measures, numerical proxies of the three developmentally-defined waves folding could not be identified.

Table 1: ANCOVA on the dependence to age and sex

Analyses of variance were conducted over the pre-term, term and post-term groups jointly to evaluate whether each parameter depends on age and sex. For each co-variable and factor, the F value is outlined with the corresponding p-value, and p-values were corrected for multiple comparisons with a FDR approach considering the 16 parameters of interest (i.e. considering each co-variable and factor independently). Statistically significant p-values suggesting linear dependence on age or sex are outlined in red (threshold $\mathrm{p}<0.05$ ).

\begin{tabular}{|c|c|c|c|c|c|c|c|}
\hline parameter $\%$ & & age & & & $\operatorname{sex}$ & & \\
\hline co-variable and factc & & $\mathrm{F}$ & $p$ & pcor & $\mathrm{F}$ & $p$ & pcor \\
\hline hemispheric volume & & 982 & 0,000 & 0,000 & 10,0 & 0,003 & 0,041 \\
\hline surface area & & 958 & 0,000 & 0,000 & 6,4 & 0,014 & 0,062 \\
\hline SI & ) & 366 & 0,000 & 0,000 & 2,5 & 0,123 & 0,152 \\
\hline AFP & & 502 & 0,000 & 0,000 & 4,8 & 0,032 & 0,074 \\
\hline \multirow[t]{3}{*}{ WL } & B4 & 630 & 0,000 & 0,000 & 6,0 & 0,018 & 0,062 \\
\hline & B5 & 634 & 0,000 & 0,000 & 5,8 & 0,019 & 0,062 \\
\hline & B6 & 633 & 0,000 & 0,000 & 5,8 & 0,019 & 0,062 \\
\hline number of parcels & B4 & 12 & 0,001 & 0,001 & 1,1 & 0,307 & 0,307 \\
\hline$\nabla$ & B5 & 104 & 0,000 & 0,000 & 4,3 & 0,042 & 0,079 \\
\hline & B6 & 274 & 0,000 & 0,000 & 4,8 & 0,032 & 0,074 \\
\hline \multirow[t]{3}{*}{ power } & B4 & 233 & 0,000 & 0,000 & 4,1 & 0,047 & 0,079 \\
\hline & B5 & 359 & 0,000 & 0,000 & 4,0 & 0,050 & 0,079 \\
\hline & B6 & 502 & 0,000 & 0,000 & 3,1 & 0,083 & 0,111 \\
\hline \multirow[t]{3}{*}{ power proportion } & B4 & 186 & 0,000 & 0,000 & 1,6 & 0,210 & 0,224 \\
\hline & B5 & 15 & 0,000 & 0,000 & 3,2 & 0,081 & 0,111 \\
\hline & B6 & 283 & 0,000 & 0,000 & 2,3 & 0,137 & 0,157 \\
\hline
\end{tabular}

Table 2: Characteristic age points

Gompertz modeling over the pre-term, term and post-term groups provided us with distinct age points (acceleration, inflection and deceleration points in weeks of PMA, with confidence intervals 
within brackets) for the sulcation index (SI), the analyzed folding power (AFP), the number of parcels for B5 and B6, and B4-6 power.

\begin{tabular}{|l|l|r|r|r|}
\hline age-points (w PMA) & acceleration & inflection & deceleration \\
\hline SI & & $25.9[25.0-26.7]$ & $33.9[33.5-34.3]$ & $43.5[42.3-44.9]$ \\
\hline AFP & & $25.1[23.9-26.2]$ & $34.7[34.1-35.3]$ & $46.0[44.3-48.3]$ \\
\hline number of parcels & B4 & - & - & - \\
\hline & B5 & $18.0[12.3-18.5]$ & $25.2[21.1-25.7]$ & $34.0[31.2-34.8]$ \\
\hline & B6 & $21.7[18.6-22.2]$ & $30.3[28.5-30.8]$ & $41.0[39.0-41.8]$ \\
\hline power & B4 & $23.3[22.2-24.2]$ & $30.6[30.3-30.9]$ & $39.4[38.4-40.6]$ \\
\hline & B5 & $26.6[25.6-27.5]$ & $34.1[33.7-34.5]$ & $43.0[41.7-44.6]$ \\
\hline & B6 & $29.0[27.6-30.4]$ & $37.7[36.9-38.7]$ & $48.1[45.0-51.7]$ \\
\hline
\end{tabular}

\section{SPANGY analyses in typical babies}

i. $\quad$ Preliminary observations: consistency of the 3 folding bands B4, B5, B6

When the total spectral power was decomposed into 7 bands according to spatial frequencies (Figure 1f), we observed that powers associated with the first four bands (B0-B3) were very similar across infants, whereas the inter-individual variability mainly relied on the last three bands (B4, B5, B6), similarly to the mature adult brain (Germanaud, et al. 2012). B4-6 wavelengths increased with age and converged to adult values in the oldest infant (Figure 3a, Tables 1, 3), suggesting that fold patterns with similar meaning and labels (e.g. B4) became larger throughout development together with the whole brain growth. In the term and post-term groups, 7 bands were clearly necessary to fully describe the folding patterns since the powers associated with B5 and B6 were high. On the reverse, in the very small brains of preterm newborns, B6 wavelengths reached both the image spatial resolution and a cut-off below which a fold might not be expected, but we chose to avoid arbitrary thresholding based on wavelengths, keeping all measures in the B6-related analyses and using a SPANGY decomposition with 7 bands as proposed for the adult brain.

B5 and B6 powers were higher than B4 power in the term and post-term groups (Figure 1f), mirroring the power spectra of adults (Germanaud, et al. 2012). On the contrary, the B4-6 power distribution was more balanced in the pre-term group, suggesting an immature spectral composition. Although power spectra could distinguish the three groups in a clear way, changes were actually continuous along age as detailed below. 
Table 3: Characteristic wavelengths for bands B4-6

Minimal and maximal values of characteristic wavelengths over the pre-term, term and postterm groups are detailed for bands B4, B5 and B6, as well as the spatial resolution expected to be covered with SPANGY. For comparison, wavelengths over ICBM adult group are reminded

\begin{tabular}{|l|l|l|l|l|}
\hline WL: $\min -\max (\mathrm{mm})$ & B4 & B5 & B6 & resolution \\
\hline pre-term & $17-34$ & $9-17$ & $4-8$ & $2-4$ \\
\hline term & $34-42$ & $17-21$ & $9-10$ & $4-5$ \\
\hline post-term & $43-55$ & $22-28$ & $11-14$ & $5-7$ \\
\hline adult & $49-64$ & $25-32$ & $12-16$ & $6-8$ \\
\hline
\end{tabular}

(Germanaud, et al. 2012).

\section{ii. $\quad$ SPANGY in the spatial domain: B4, B5, B6 associated sulci elements (parcels)}

Over the 27-62w period, we observed an anatomically coherent evolution of the segmentation of cortical sulci with SPANGY according to their frequency components. B4associated elements were present in all newborns, overlapping the main and earliest folds, whereas elements associated with B5 then B6 increased with age following the appearance of branches, twists and variable folds (Figure 2d,e). This suggested that elements associated with B4, B5 and B6 might match substantially with primary, secondary and tertiary folds respectively, as proposed in the healthy adult brain (Germanaud, et al. 2012) and as comforted by observations of gyral simplification in severe microcephalies (Germanaud, et al. 2014).

This was further quantified based on the number of sulci elements. Whereas the number of B4 parcels remained constant with age (mean number $~ 22.5 \pm 2.6$ ), the numbers of B5 and B6 parcels increased dramatically (Table 1) and non-linearly (Figure 3b). Gompertz modeling highlighted that these numbers decelerated around 34w and 41w PMA for B5 and B6 respectively (Table 2), and reached a plateau around term age and during early infancy.

Figure 3: Evolution of SPANGY parameters in the three typical groups

The characteristic wavelength (a), number of parcels (b), power (c) and power proportion (d) are presented for bands B4 (deep blue), B5 (light blue) and B6 (green) as a function of the newborns postmenstrual age. B4-B6 proportions are also represented in 3D with ellipsoids highlighting the $4 \mathrm{k}$ means clusters (e). Regarding the power law between cortical surface and brain size $\left(S=b V^{a}\right)$, different scaling exponents $a$ were observed in these 4 clusters (f).

The dots code for the group (see Figure 1). For the numbers of parcels (b) and powers (c), the curves outline the Gompertz models, while in (d) they correspond to ratios between Gompertz models to highlight each band power proportions. 


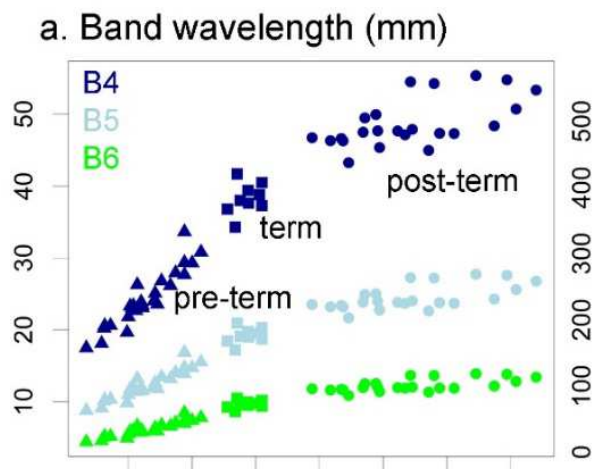

$\begin{array}{lllllll}30 & 35 & 40 & 45 & 50 & 55 & 60\end{array}$

c. Band power b. Band number of parcels

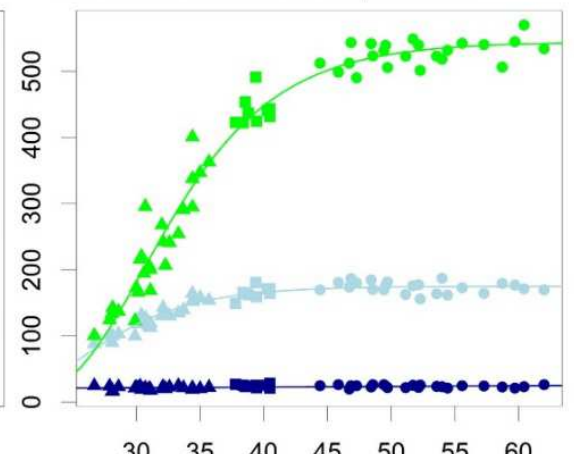

d. Band proportions (power)

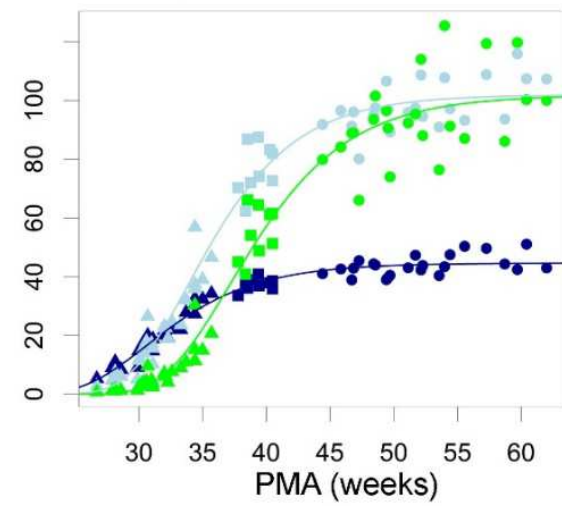

e. Clustering on band proportions f. Power law $S=b V^{a}$
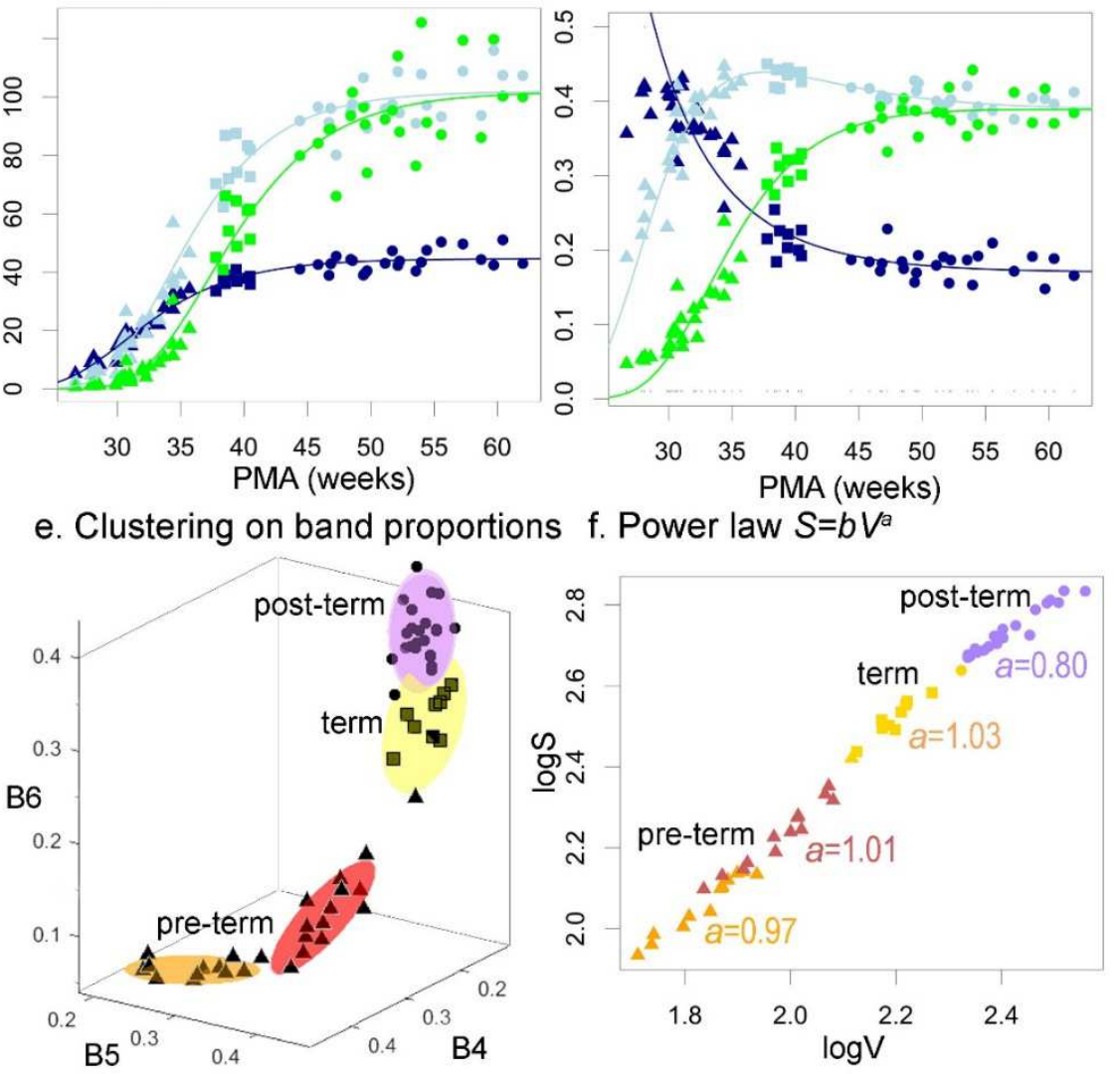

iii. $\quad$ SPANGY in the spectral domain: B4, B5, B6 spectral powers

Coherently with AFP, B4 to B6 powers increased over the considered period (Figure $3 \mathrm{c}$, Table 1), with a different time course across the bands (Figure 3c). Regarding age points measured with Gompertz modeling (Table 2), acceleration was detected between 23w (B4) and 29w (B6), and inflexion between 31w (B4) and 38w (B6), highlighting an intense folding process during the pre-term period. Asymptotic deceleration was detected between $39 \mathrm{w}$ (B4) and $48 \mathrm{w}$ (B6), reflecting that the folding intensity reached a plateau during early infancy. These results suggested that the 3 bands captured successive waves of folding. 
Furthermore, the power proportions changed dramatically from the pre-term to the post-term period (Figure 3d). While pre-term newborns showed high B4 proportion, full-term newborns and infants showed adult-like spectral composition with high B5 and B6 proportions. From around 30w, proportions continuously decreased for B4 and increased for B6, reaching plateaus during the late pre-term to early term period. B5 proportion evolution was more complex, with a transient peak around 37w suggestive of an apex of the secondary folding wave. In summary, the folding course seemed to be characterized by the dynamic enrichment of the curvature pattern in B4 to B6 spatial frequencies.

These observations were further synthetized by specifying developmental sub-periods with distinct folding dynamic features. All infants were clustered using kmeans algorithm applied to the complementary B4-6 power proportions, which provided a reliable separation in terms of age, with few overlaps across the 4 clusters (Figure 3e). The first and second clusters gathered almost all pre-term newborns over 2 age sub-periods: from $27 \mathrm{w}$ to $31 \mathrm{w}$ and from $31 \mathrm{w}$ to $36 \mathrm{w}$, coherently with the appearance of secondary folds around $32 \mathrm{w}$ PMA. The third cluster gathered all full-term newborns, one single pre-term newborn at 34w PMA and one $47 \mathrm{w}$-PMA infant, in relative agreement with the appearance of tertiary folds around term age. The fourth cluster gathered all the other infants, for whom the folding process had slowed down. Considering 3 clusters instead of 4 , the two latter clusters were joined, with no change in the two former ones. As a whole, these results were in line with the previous description of three successive waves of folding in fetus specimen (Chi, et al. 1977).

In each cluster, cortical surface area and hemispheric volume were linearly related on a log-scale (Figure 3f). The measured scaling exponent of this allometric relationship appeared to decrease throughout development: $a=0.97-1.03$ in the first three clusters, versus $a=0.80$ in the fourth cluster. We observed a similar developmental trend when grouping the two pre-term clusters $(a=1.09)$, and the term/post-term clusters $(a=0.96)$.

\section{Atypical folding in prematurely born infants at TEA}

Similarly to the pre-term, term and post-term groups, SPANGY analyses were conducted in all premature infants at TEA based on images and segmentations all passing the manual quality check (Figure 4). Cortical reconstructions and spectral segmentations of sulci showed no obvious difference between the TEA and full-term newborns. Based on quantitative comparisons of parameters (Figure 5, Table 4), we found no deviations for hemispheric volume nor cortical surface area in these 10 TEA infants compared with typical developmental trajectories, suggesting that brain growth abnormalities were absent or too 
small to be detected given our statistical power. However, the global folding intensity (sulcation index and AFP) was lower, mainly because of a significant reduction in B4 power (Table 4, see effect sizes in supplementary Table S2). Eventually, we found no difference in B4-6 parcels numbers nor in power proportions (Supplementary Information \#2), suggesting that the fold numbers were barely affected.

Figure 4: Visual comparison of full-term newborns versus premature infants at TEA

As in Figure 1, T2w images (a), segmentations of the cortical boundary (b), 3D reconstructions of the inner cortical surface (c) and SPANGY segmentations (d) are outlined for 2 fullterms (39.4w and 38.5w PMA) and 2 preterms at TEA (41w, 40.5w PMA) (all boys). The zooms on the central sulcus (e) suggest simpler folding patterns in premature infants compared with full-term newborns despite equivalent hemispheric volumes ( 333 versus $334 \mathrm{~mL}, 371$ versus $379 \mathrm{~mL}$ ).

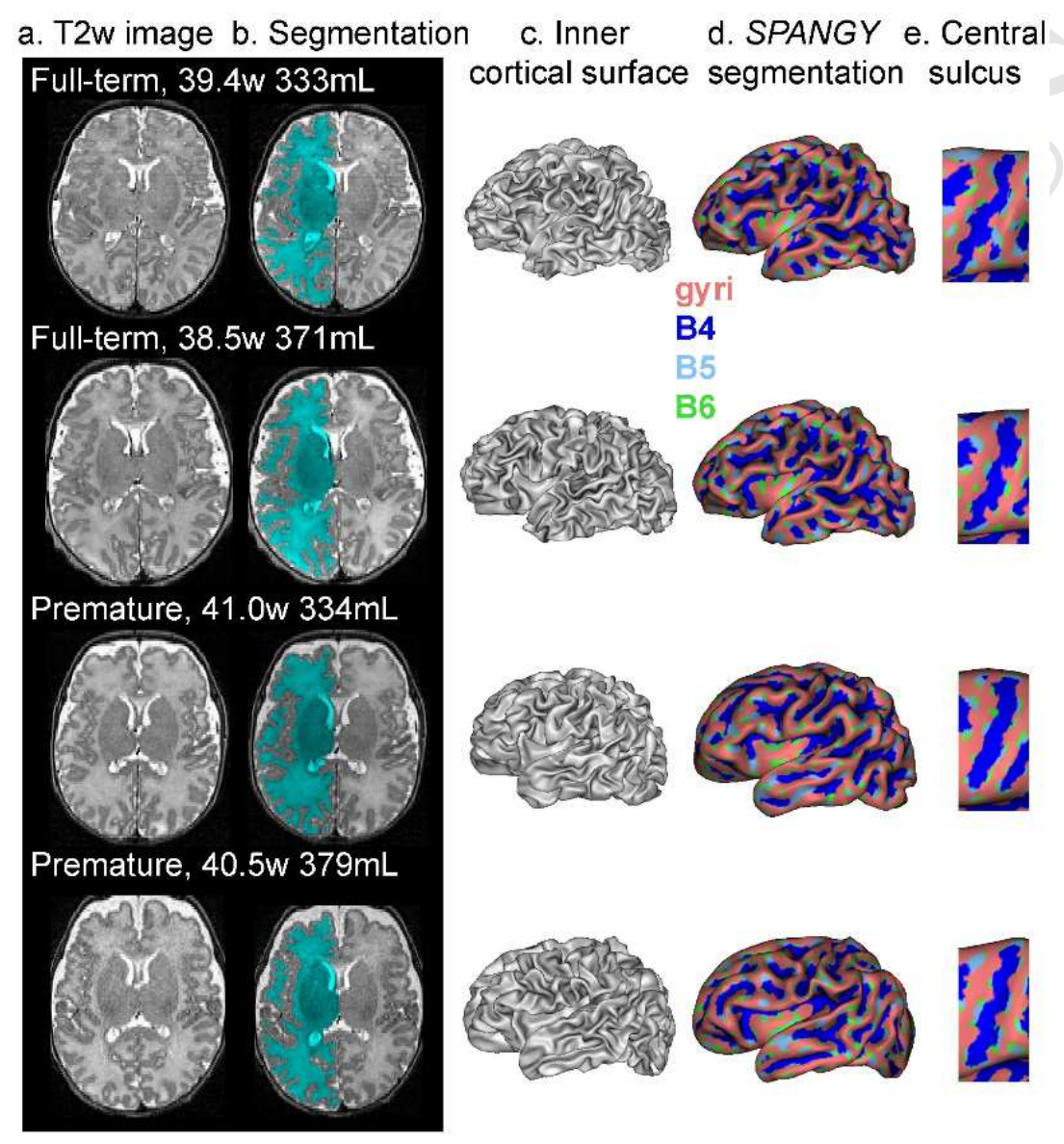


Figure 5: Atypical cortical folding in premature infants at TEA

Whole-brain parameters (a: hemispheric volume, cortical surface area, sulcation index) and B4-6 bands characteristics (b: number of parcels, c: power, d: power proportion) are presented as a function of PMA for premature infants at TEA (in black) compared with babies of the pre-term, term and post-term groups (in grey). Premature infants at TEA clearly deviate from the typical folding trajectory (Gompertz models from Figures 1 and 3 are outlined by grey curves), for the sulcation index and B4 power (see also Table 4).

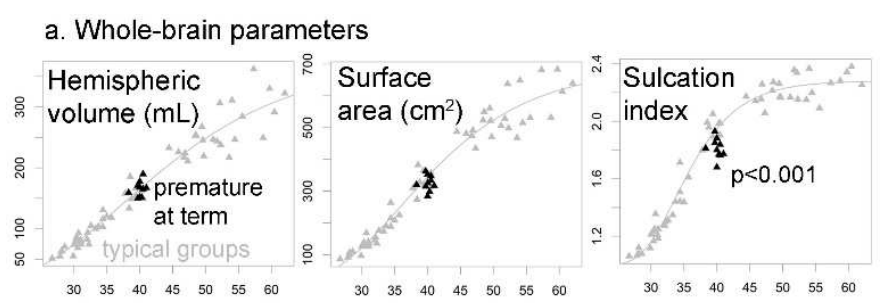

b. Band number of parcels
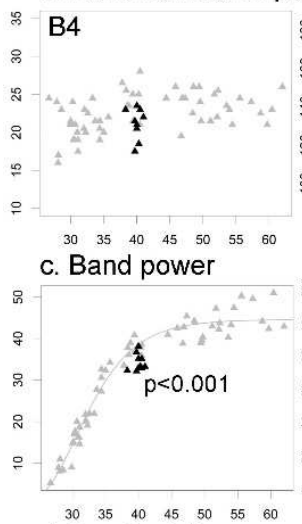

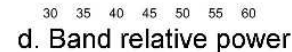

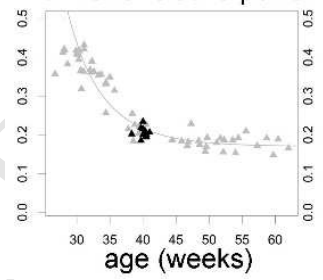

B5

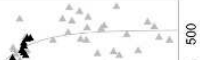

premature at term
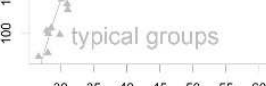

$\begin{array}{llllllll}30 & 35 & 40 & 45 & 50 & 55 & 60\end{array}$
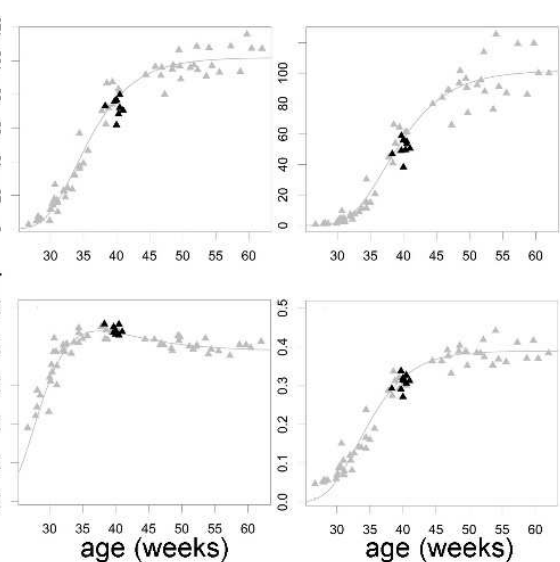

B6
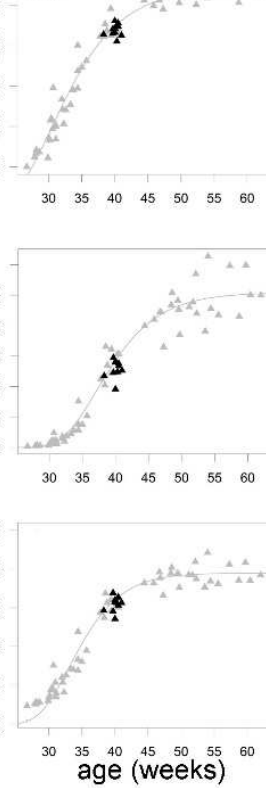

Table 4: Deviations in folding development in premature infants at TEA

For each parameter, we performed shuffle and split (S\&S) analysis to compare the group of premature infants at TEA with the typical trajectories of pre-term, term and postterm infants (significant $\mathrm{p}$-values are outlined in red for threshold $\mathrm{p}<0.05$, and with $*$ for $\mathrm{p}<0.005$ corresponding to Bonferroni correction for 10 comparisons). Results suggested deviations in the folding properties, particularly related to lower B4 power. Results from paired $\mathrm{T}$ tests, notably for power proportions, are provided in Supplementary Information \#2.

\begin{tabular}{|l|l|r|}
\hline Difference between & \multicolumn{1}{|l|}{ S\&S analyses } \\
\cline { 3 - 3 } TEA and typical groups & $\mathrm{p}$ \\
\hline hemispheric volume & & 0,742 \\
\hline surface area & & 0,067 \\
\hline SI & & $<0,001 *$ \\
\hline AFP & & $0,005 *$ \\
\hline number of parcels & B4 & 0,118 \\
\hline & B5 & 0,414 \\
\hline power & B6 & 0,127 \\
& B4 & $<0,001 *$ \\
& B5 & 0,009 \\
\hline & B6 & 0,030 \\
\hline
\end{tabular}
Abbreviations: AFP analyzed folding power, SI sulcation index. 


\section{Discussion}

In this study, we analyzed the dynamics of cortical folding over a crucial developmental period in a sample of pre-term, full-term newborns and infants. Although we considered cross-sectional data over limited groups of subjects, we could highlight different folding waves that were captured and quantified at the individual level with spectral analysis of brain curvature. We further showed that the SPANGY approach is sensitive enough to detect and locate subtle deviations in a small group of premature infants at TEA while they showed no major anomaly in brain growth.

\section{SPANGY: an approach allowing to map and quantify the folding waves}

Like in the adult brain (Germanaud, et al. 2012), we demonstrate that the patterns of cortical surface curvature in the developing brain were meaningfully depicted by bands of increasing spatial frequencies, specifically bands B4, B5 and B6. In comparison with previous studies, SPANGY characterizes the sulci patterns quantitatively without supervision of folds localization. Our study supports the relevance of frequency-based descriptions of the folding sequence throughout development. We found characteristic age-related inflexions in the developmental increase of B4 to B6 spectral powers and of number of parcels. B4-related sulci elements were detected early on in all preterms, and their corresponding number of parcels did not evolve with age, in analogy with primary folds. The increase in B4 power throughout development was mainly dependent on the surface increase related to brain growth. Our clustering approach based on B4-6 power proportions suggested a relevant inflexion around 31w PMA with decreasing B4 contribution and increasing B5 contribution while B6 was still anecdotic. The majority of B5-related sulcus elements appeared in a second folding wave with a relative peak around 37w PMA, in good morphological analogy with secondary folds for most of them. After 38w PMA, two additional sub-periods were outlined, matching first a significant decrease in B5 contribution from term age due to B6 dynamics, and second, the end of the curvature pattern enrichment a few weeks later in infancy. Such results strongly support the link proposed in the adult brain between the three types of B4-6 sulci elements and the three successive developmentally-defined waves of folding. However, the anatomical correspondence of B5 and B6 with secondary and tertiary waves remained somewhat imperfect, with earlier acceleration age points than expected. These discrepancies should be discussed in light of the often neglected overlap between folding waves, notably in relation to folding heterochrony across cortical lobes and regions (Chi, et al. 1977). 
Alternatively, several issues can be discussed. Firstly, we did not have other option than considering preterm newborns at birth to define typical developmental trajectories $e x$ utero, whereas preterm birth per se is not a normal condition for brain growth. But conversely, it would not be relevant to include MRI measures obtained from healthy fetuses, since we recently demonstrated that the transition from intra- to extra-uterine environment in itself has a major impact on folding properties (Lefèvre, et al. 2016). The CSF environment and the cortical configurations seem to differ strongly in pre- and post-natal brains, so mixing information from fetuses and full-term neonates and infants would not provide a continuous description of the folding progression from the pre-term to the post-term periods. At the methodological level, folding patterns might tend to merge in small brains due to potential spatial noise affecting cortical surface segmentations. Second, mismatches might also outline some limits of the SPANGY model. Sulcal and gyral domains are treated together while they are not strictly symmetrical in shape (Germanaud, et al. 2012). Moreover, SPANGY being a Fourier-like strategy, it uses higher frequencies (accounting for B6-related parcels) for both new pattern depiction (real tertiary sulci elements) and depiction of sharpness (angular sulcal edges for instance). Dealing with these last two flaws would be possible by using more localized spectral procedures such as wavelet analysis (Yu, et al. 2007).

To confirm and clarify the extent of the anatomical analogy between B4-6 sulci elements and the successive folding waves, it would be essential to test SPANGY analyses on ex utero longitudinal data at different ages when brains show different progression of primary, secondary and tertiary foldings. This would be the only way to definitely decipher the influence of developmental processes on SPANGY patterns while taking into account the inter-subject variability that might have blurred our findings. Nevertheless, longitudinal analyses will not be straightforward, as they will require reliable registration between brains of different size and folding complexity, which is also a major methodological issue. Also because our groups had limited sizes and unbalanced numbers over the different age spreads, this study as a whole should be considered as a first step to describe the progression of folding over a crucial developmental period. It has to be pursued by the analysis of large-scale longitudinal data that start to become available for the scientific community.

Since the SPANGY approach relies on the analysis of cortical curvature, our results crucially depend on accurate inner cortical surface segmentation. While motion-related artifacts are often visible on brain images of unsedated infants, here we only analyzed T2w images that were relatively free from such artifacts, which required to select adequate data 
among the ones acquired. Applying our approach to clinical populations would require to correct images for motion artefacts observed within slices and across slices, before further analyses. In this study, particular care was taken to minimize errors and possible biases across individuals and across groups.

Nevertheless, we have to mention several possible issues. In this study, differences in image acquisition (MRI scanner types, strength, coil, image spatial resolution in the three directions) and post-processing (segmentation pipelines) between the pre-term group versus the term and post-term groups might have impacted our quantitative measures. To our disadvantage, acquiring MRI data in such populations is challenging and requires a long inclusion period (potential system changes), particularly for preterm newborns (without neurological complications and imaged in the first days after birth), and for healthy infants (imaged without sedation). Yet, the image quality was relatively similar across groups: despite the lower acquisition settings in preterms, the signal-to-noise ratio of $\mathrm{T} 2 \mathrm{w}$ images was similar because of the higher brain water content, and motion artifacts were not different thanks to equivalent acquisition times (fewer slices were required in preterms). Indeed, images showed some variability across subjects in relation to the brain maturational properties, but the grey-white matter contrast was in any case adequate for the segmentation process (Figure 2a-c). And interactive manual corrections were performed in erroneous places, mostly where the progressing myelination blurred the contrast in infants, but rarely because of motion artifacts. A single operator (JD) reviewed the consistency of segmentations across all newborns. We assumed sufficient reliability of our results across groups in view of the two indirect following arguments. First, whereas another research group reported a trend toward lower folding measured from $1.5 \mathrm{~T}$ images compared with $3 \mathrm{~T}$ between $26 \mathrm{w}$ and $40 \mathrm{w}$ PMA (Kim, et al. 2016a), we observed a strong continuity in morphometric and SPANGY parameters across ages with our tools. Secondly, the kmeans clustering used to define developmental sub-periods was quite insensitive to differences in acquisition and postprocessing characteristics: 2 clusters reliably split the pre-term group whatever the number of clusters (3 or 4$)$.

In light of these indirect arguments, we expect that our typical developmental trajectories were little biased by acquisition and post-processing differences across age groups. And we believe that the SPANGY approach can be applied reliably on inner cortical surfaces generated with alternative segmentation methods, as proposed by other groups in fetuses, preterms and infants (e.g. (Clouchoux, et al. 2012; Gui, et al. 2012; Hill, et al. 2010; 
Li, et al. 2014a; Makropoulos, et al. 2015; Moeskops, et al. 2015)). Nevertheless, we will not be able to dispense of replication studies to confirm these expectations, particularly when databases acquired with similar settings over the whole developmental period will become available. Future works will also be required to investigate whether the SPANGY measures might be influenced by factors related to image acquisition and surface extraction, and to validate their reliable use as imaging biomarkers in clinical applications as proposed for sulcal pits (Im, et al. 2013).

\section{Investigating the typical process of cortical folding in the developing brain}

For the first time, the SPANGY approach provided a quantitative description of the successive folding waves ex utero, continuously over the late pre-term and early post-term periods. Our results are particularly informative at several levels. First, non-linear developmental trajectories were outlined, in agreement with previous studies in fetuses (Wright, et al. 2014) and infants (Li, et al. 2014b). Interestingly, a differential progression of folding was revealed within the pre-term period, with a slow beginning and a secondary progression from around $31 \mathrm{w}$, followed by smaller changes from term onwards and during early infancy. Besides age, we observed some inter-individual variability across infants in relation to sex, in agreement with a recent study reporting folding differences between boys and girls during the first 2 years ( $\mathrm{Li}$, et al. 2014b). But as demonstrated in adults (Germanaud, et al. 2012) and suggested in infants (Li, et al. 2014b), differences in brain volume accounted for this sex effect (Supplementary Information \#3).

As for allometric relationships between cortical surface area and brain volume, the scaling exponent measured over the two pre-term clusters jointly ( $a=1.09$ ) was a bit lower than previously reported measures in preterm newborns $(a=1.25-1.33)$ (Kapellou, et al. 2006; Paul, et al. 2014). On the other hand, the exponent over the two term/post-term clusters $(a=0.96)$ was similar to the adult one (Germanaud, et al. 2012). This suggests that the cortical extension going with brain size is more intense during the pre-term period in relation to age and early growth (developmental allometry) than after birth when it reaches a rather adult-like value related to inter-individual variability independently of growth (static allometry due to size polymorphism, in relation to sex, genetic and nutritional factors).

Interpreting the neurobiological substrates of folding increase remains a major challenge since this process is supposed to rely on several intermingled mechanisms in the cortex at both the microstructural and morphological levels. The strong folding increase between 29 and 39w PMA might correspond to the expansion of cortical tissue related to the 
migration of subplate neurons into the cortex, with successive waves triggering the primary, secondary and tertiary foldings. With this in mind, evaluating differences in folding progression across brain regions would be an interesting approach, since age-related spatial gradients of folding and of subplate regression have been reported recently. This would be the scope of additional analyses, based on local parameters instead of global hemispheric measures. Investigating the possible links between cortical folding, thickness ( $\mathrm{Li}$, et al. 2015) and microstructure (Ball, et al. 2013) would be a valuable perspective to decipher which microstructural or mechanical mechanisms might drive the folding process. In that respect, SPANGY parameters might constitute relevant references for theoretical and experimental modeling of early cortical folding.

\section{Mapping atypical folding in premature infants imaged at TEA}

To investigate whether the SPANGY approach would be sensitive enough to detect and locate subtle and specific deviations of the folding process, we also studied a group of premature infants at TEA, without major brain anomaly contrarily to previous studies (Engelhardt, et al. 2015; Makropoulos, et al. 2015). These preterms were comparable to each typical group in regards to different growth characteristics, as they had similar duration of pre-natal growth than the pre-term group, of total growth than the term group, and of postnatal growth than the post-term group. Although we did not detect alterations in brain growth (cortical volume and surface area within the normal ranges), our approach showed that preterms at TEA had lower global folding power than typical infants, mainly associated with lower B4 power. This suggests that folds developing early on, at the time of preterm birth, were the most impacted. Nevertheless, we cannot conclude on the directionality of these findings: deviations in primary folding might either result from prematurity and post-natal growth, or reflect intra-uterine disturbances leading to premature birth and further atypical folding. Decreases in B5 and B6 powers were less significant (Table 4), and might rather reflect the impact of ex utero growth on an immature brain born pre-term. These results highlight SPANGY sensitivity to detect subtle folding deviations.

Although we considered a rather small group of preterm infants at TEA, our findings on the differential impact of prematurity on the successive folding waves are partly consistent with the few previous studies. The gyrification index seemed to decrease with increasing abnormality score at 30w and 40w PMA (Moeskops, et al. 2015), however this study did not take into account possible inter-individual differences in brain size. We also previously showed that sulci developing earliest (e.g. the insula, central and superior temporal sulci) are 
the most affected at 30w and 40w PMA by adverse clinical factors (e.g. birth weight) (Kersbergen, et al. 2016). Future studies in larger cohorts of preterms and with longitudinal follow-up will be required to investigate the links between folding impairments and clinical factors related to pregnancy and in utero growth (e.g. multiple pregnancy, birth weight, intrauterine growth restriction) (Dubois, et al. 2008a; Kersbergen, et al. 2016), environmental sensory exposure (Pineda, et al. 2014), or functional outcome during toddlerhood (Kersbergen, et al. 2016; Spann, et al. 2014).

\section{Conclusion}

This study was a first step to describe the normal progression of primary, secondary and tertiary folds over a crucial developmental period ex utero, which have to be pursued by the analysis of large-scale longitudinal data. We further highlighted the potential and sensitivity of SPANGY to detect subtle folding deviations by comparing infants differing in the amount of intra- versus extra-uterine growth. We suggested that this approach could provide the first quantitative and objective measure of the folding stage at the individual brain level, which might be crucial in the prospect of individual predictions in several developmental diseases (Mangin, et al. 2010) particularly with brain growth insufficiency and microcephaly (Germanaud, et al. 2014).

\section{Acknowledgements}

The authors thank the unit of child growth in Geneva University Hospitals (Drs S. Sizonenko, R. Ha Vinh Leuchter, L. Gui) for precious help in scanning the newborns and preparing the data. They also thank the UNIACT clinical team of NeuroSpin and P. Adibpour for precious help in scanning the infants and checking the image quality.

This work was supported by the Fyssen Foundation, the "Fondation de France", the Paris Neurosciences School (ENP), the French National Agency for Research (ANR-12-JS03-00101, MODEGY), the Geneva CIBM imaging center, the Swiss National Science Foundation, the Leenards Foundation, the European consortium NEOBRAIN (LSHM-CT-2006-036534), and the European Union's Horizon 2020 Framework Programme for Research and Innovation under Grant Agreement No. 604102 (HBP's ramp-up phase). 


\section{References}

Awate SP, Yushkevich P, Licht D, Gee JC. 2009. Gender differences in cerebral cortical folding: multivariate complexity-shape analysis with insights into handling brain-volume differences. Med Image Comput Comput Assist Interv 12(Pt 2):200-7.

Ball G, Srinivasan L, Aljabar P, Counsell SJ, Durighel G, Hajnal JV, Rutherford MA, Edwards AD. 2013. Development of cortical microstructure in the preterm human brain. Proc Natl Acad Sci U S A 110(23):9541-9546.

Chi JG, Dooling EC, Gilles FH. 1977. Gyral development of the human brain. Ann Neurol 1(1):86-93.

Clouchoux C, Kudelski D, Gholipour A, Warfield SK, Viseur S, Bouyssi-Kobar M, Mari JL, Evans AC, du Plessis AJ, Limperopoulos C. 2012. Quantitative in vivo MRI measurement of cortical development in the fetus. Brain Struct Funct 217(1):127-39.

Dubois J, Benders M, Borradori-Tolsa C, Cachia A, Lazeyras F, Ha-Vinh Leuchter R, Sizonenko SV, Warfield SK, Mangin JF, Huppi PS. 2008a. Primary cortical folding in the human newborn: an early marker of later functional development. Brain 131(Pt 8):2028-41.

Dubois J, Benders M, Cachia A, Lazeyras F, Ha-Vinh Leuchter R, Sizonenko SV, Borradori-Tolsa C, Mangin JF, Huppi PS. 2008b. Mapping the early cortical folding process in the preterm newborn brain. Cereb Cortex 18(6):1444-54.

Dubois J, Benders M, Lazeyras F, Borradori-Tolsa C, Leuchter RH, Mangin JF, Huppi PS. 2010. Structural asymmetries of perisylvian regions in the preterm newborn. Neuroimage 52(1):32-42.

Dubois J, Dehaene-Lambertz G. 2015. Fetal and postnatal development of the cortex: MRI and genetics. In: Arthur W. Toga, editor, Brain Mapping: An Encyclopedic Reference, Academic press: Elsevier 2:1119.

Dubois J, Dehaene-Lambertz G, Kulikova S, Poupon C, Hüppi PS, Hertz-Pannier L. 2014. The early development of brain white matter: a review of imaging studies in fetuses, newborns and infants. Neuroscience 276(C):48-71.

Engelhardt E, Inder TE, Alexopoulos D, Dierker DL, Hill J, Van Essen D, Neil JJ. 2015. Regional Impairments of Cortical Folding in Premature Infants. Ann Neurol 77(1):154-62.

Feess-Higgins A, Laroche JC. 1987. Development of the human foetal brain: an anatomical atlas. Inserm-CNRS, Masson.

Fernandez V, Llinares-Benadero C, Borrell V. 2016. Cerebral cortex expansion and folding: what have we learned? EMBO J 35(10):1021-1044.

Fischer C, Operto G, Laguitton S, Perrot M, Denghien I, Rivière D, Mangin JF. 2012. Morphologist 2012: the new morphological pipeline of BrainVISA. Proceedings of the $18^{\text {th }}$ HBM Scientific Meeting, Beijing, China. NeuroImage:670.

Germanaud D, Lefevre J, Fischer C, Bintner M, Curie A, des Portes V, Eliez S, Elmaleh-Berges M, Lamblin D, Passemard S and others. 2014. Simplified gyral pattern in severe developmental microcephalies? New insights from allometric modeling for spatial and spectral analysis of gyrification. Neuroimage $102 \mathrm{Pt}$ 2:317-31.

Germanaud D, Lefèvre J, Toro R, Fischer C, Dubois J, Hertz-Pannier L, Mangin JF. 2012. Larger is twistier: spectral analysis of gyrification (SPANGY) applied to adult brain size polymorphism. Neuroimage 63(3):1257-72.

Girard NJ, Chaumoitre K. 2012. The brain in the belly: what and how of fetal neuroimaging? J Magn Reson Imaging 36(4):788-804.

Gui L, Lisowski R, Faundez T, Huppi PS, Lazeyras F, Kocher M. 2012. Morphology-driven automatic segmentation of MR images of the neonatal brain. Med Image Anal 16(8):1565-79.

Habas PA, Scott JA, Roosta A, Rajagopalan V, Kim K, Rousseau F, Barkovich AJ, Glenn OA, Studholme C. 2012. Early folding patterns and asymmetries of the normal human brain detected from in utero MRI. Cereb Cortex 22(1):13-25.

Hill J, Dierker D, Neil J, Inder T, Knutsen A, Harwell J, Coalson T, Van Essen D. 2010. A surface-based analysis of hemispheric asymmetries and folding of cerebral cortex in term-born human infants. J Neurosci 30(6):2268-76.

Im K, Lee JM, Jeon S, Kim JH, Seo SW, Na DL, Grant PE. 2013. Reliable identification of deep sulcal pits: the effects of scan session, scanner, and surface extraction tool. PLoS One 8(1):e53678.

Kabdebon C, Leroy F, Simmonet H, Perrot M, Dubois J, Dehaene-Lambertz G. 2014. Anatomical correlations of the international 10-20 electrode placement system in infants. Neuroimage 99:342-356. 
Kapellou O, Counsell SJ, Kennea N, Dyet L, Saeed N, Stark J, Maalouf E, Duggan P, Ajayi-Obe M, Hajnal J and others. 2006. Abnormal cortical development after premature birth shown by altered allometric scaling of brain growth. PLoS Med 3(8):e265.

Kersbergen KJ, Leroy F, Išgum I, Groenendaal F, de Vries LS, Claessens NHP, van Haastert IC, Moeskops P, Fischer C, Mangin JF and others. 2016. Relation between clinical risk factors, early cortical changes, and neurodevelopmental outcome in preterm infants. Neuroimage 142:301-310.

Kim H, Lepage C, Maheshwary R, Jeon S, Evans AC, Hess CP, Barkovich AJ, Xu D. 2016a. NEOCIVET: Towards accurate morphometry of neonatal gyrification and clinical applications in preterm newborns. Neuroimage 138:28-42.

Kim SH, Lyu I, Fonov VS, Vachet C, Hazlett HC, Smith RG, Piven J, Dager SR, McKinstry RC, Pruett JR, Jr. and others. 2016b. Development of cortical shape in the human brain from 6 to 24months of age via a novel measure of shape complexity. Neuroimage 135:163-176.

Lambert RJ, Mytilinaios I, Maitland L, Brown AM. 2012. Monte Carlo simulation of parameter confidence intervals for non-linear regression analysis of biological data using Microsoft Excel. Comput Methods Programs Biomed 107(2):155-63.

Lefèvre J, Auzias G. 2015. Spherical Parameterization for Genus Zero Surfaces Using Laplace-Beltrami Eigenfunctions. Geometric Science of Information 9389:121-129.

Lefèvre J, Auzias G, Germanaud D. 2014. Brain lobes revealed by spectral clustering. IEEE International Conference on Pattern Recognition:562-567.

Lefèvre J, Germanaud D, Dubois J, Rousseau F, de Macedo Santos I, Angleys H, Mangin JF, Huppi PS, Girard N, De Guio F and others. 2016. Are Developmental Trajectories of Cortical Folding Comparable Between Cross-sectional Datasets of Fetuses and Preterm Newborns? Cereb Cortex 26(7):3023-35.

Lefèvre J, Germanaud D, Fischer C, Toro R, Rivière D, Coulon O. 2012. Fast Surface-based Measurements using First Eigenfunction of the Laplace-Beltrami Operator: interest for sulcal description. IEEE International Symposium on Biomedical Imaging (ISBI) DOI: 10.1109/ISBI.2012.6235863.

Lefèvre J, Mangin JF. 2010. A reaction-diffusion model of human brain development. PLoS Comput Biol 6(4):e1000749.

Leroy F, Mangin JF, Rousseau F, Glasel H, Hertz-Pannier L, Dubois J, Dehaene-Lambertz G. 2011. Atlas-free surface reconstruction of the cortical grey-white interface in infants. PLoS One 6(11):e27128.

Li G, Lin W, Gilmore JH, Shen D. 2015. Spatial Patterns, Longitudinal Development, and Hemispheric Asymmetries of Cortical Thickness in Infants from Birth to 2 Years of Age. J Neurosci 35(24):9150-62.

Li G, Nie J, Wang L, Shi F, Gilmore JH, Lin W, Shen D. 2014a. Measuring the dynamic longitudinal cortex development in infants by reconstruction of temporally consistent cortical surfaces. Neuroimage 90:266-79.

Li G, Wang L, Shi F, Lyall AE, Lin W, Gilmore JH, Shen D. 2014b. Mapping longitudinal development of local cortical gyrification in infants from birth to 2 years of age. J Neurosci 34(12):4228-38.

Luecke RH, Wosilait WD, Young JF. 1995. Mathematical representation of organ growth in the human embryo/fetus. Int J Biomed Comput 39(3):337-47.

Makropoulos A, Aljabar P, Wright R, Huning B, Merchant N, Arichi T, Tusor N, Hajnal JV, Edwards AD, Counsell SJ and others. 2015. Regional growth and atlasing of the developing human brain. Neuroimage 125:456-478.

Mangin JF, Jouvent E, Cachia A. 2010. In-vivo measurement of cortical morphology: means and meanings. Curr Opin Neurol 23(4):359-67.

Mangin JF, Riviere D, Cachia A, Duchesnay E, Cointepas Y, Papadopoulos-Orfanos D, Scifo P, Ochiai T, Brunelle F, Regis J. 2004. A framework to study the cortical folding patterns. Neuroimage 23 Suppl $1:$ S129-38.

Melbourne A, Kendall GS, Cardoso MJ, Gunny R, Robertson NJ, Marlow N, Ourselin S. 2014. Preterm birth affects the developmental synergy between cortical folding and cortical connectivity observed on multimodal MRI. Neuroimage 89:23-34.

Meng Y, Li G, Lin W, Gilmore JH, Shen D. 2014. Spatial distribution and longitudinal development of deep cortical sulcal landmarks in infants. Neuroimage 100:206-18.

Ment LR, Hirtz D, Huppi PS. 2009. Imaging biomarkers of outcome in the developing preterm brain. Lancet Neurol 8(11):1042-55.

Mischan MM, Zambello de Pinho S, de Carvalho LR. 2011. Determination of a point sufficiently close to the asymptote in nonlinear growth functions. Scientia Agricola 68(1):109-114.

Moeskops P, Benders MJ, Kersbergen KJ, Groenendaal F, de Vries LS, Viergever MA, Isgum I. 2015. Development of Cortical Morphology Evaluated with Longitudinal MR Brain Images of Preterm Infants. PLoS One 10(7):e0131552. 
Orasanu E, Melbourne A, Cardoso MJ, Lomabert H, Kendall GS, Robertson NJ, Marlow N, Ourselin S. 2016. Cortical folding of the preterm brain: a longitudinal analysis of extremely preterm born neonates using spectral matching. Brain Behav:e00488.

Padilla N, Alexandrou G, Blennow M, Lagercrantz H, Aden U. 2015. Brain Growth Gains and Losses in Extremely Preterm Infants at Term. Cereb Cortex 25(7):1897-905.

Paul RA, Smyser CD, Rogers CE, English I, Wallendorf M, Alexopoulos D, Meyer EJ, Van Essen DC, Neil JJ, Inder TE. 2014. An allometric scaling relationship in the brain of preterm infants. Ann Clin Transl Neurol 1(11):933-7.

Pineda RG, Neil J, Dierker D, Smyser CD, Wallendorf M, Kidokoro H, Reynolds LC, Walker S, Rogers C, Mathur AM and others. 2014. Alterations in brain structure and neurodevelopmental outcome in preterm infants hospitalized in different neonatal intensive care unit environments. J Pediatr 164(1):52$60 \mathrm{e} 2$.

Rajagopalan V, Scott J, Habas PA, Kim K, Corbett-Detig J, Rousseau F, Barkovich AJ, Glenn OA, Studholme C. 2011. Local tissue growth patterns underlying normal fetal human brain gyrification quantified in utero. J Neurosci 31(8):2878-87.

Rodriguez-Carranza CE, Mukherjee P, Vigneron D, Barkovich J, Studholme C. 2008. A framework for in vivo quantification of regional brain folding in premature neonates. Neuroimage 41(2):462-78.

Shimony JS, Smyser CD, Wideman G, Alexopoulos D, Hill J, Harwell J, Dierker D, Van Essen DC, Inder TE, Neil JJ. 2015. Comparison of cortical folding measures for evaluation of developing human brain. Neuroimage 125:780-790.

Spann MN, Bansal R, Rosen TS, Peterson BS. 2014. Morphological features of the neonatal brain support development of subsequent cognitive, language, and motor abilities. Hum Brain Mapp 35(9):4459-74.

Toro R, Perron M, Pike B, Richer L, Veillette S, Pausova Z, Paus T. 2008. Brain size and folding of the human cerebral cortex. Cereb Cortex 18(10):2352-7.

Van Essen DC. 1997. A tension-based theory of morphogenesis and compact wiring in the central nervous system. Nature 385(6614):313-8.

Welker W. 1990. Why does cerebral cortex fissure and fold? A review of determinants of gyri and sulci. . In: Comparative structure and evolution of cerebral cortex. Jones E, Peters A eds. Plenum, New York. Part II, vol 8B:3-136.

Wosilait WD, Luecke RH, Young JF. 1992. A mathematical analysis of human embryonic and fetal growth data. Growth Dev Aging 56(4):249-57.

Wright R, Kyriakopoulou V, Ledig C, Rutherford MA, Hajnal JV, Rueckert D, Aljabar P. 2014. Automatic quantification of normal cortical folding patterns from fetal brain MRI. Neuroimage 91:21-32.

Yu P, Yeo BT, Grant PE, Fischl B, Golland P. 2007. Cortical Folding Development Study based on OverComplete Spherical Wavelets. Proc IEEE Int Conf Comput Vis 2007.

Zilles K, Palomero-Gallagher N, Amunts K. 2013. Development of cortical folding during evolution and ontogeny. Trends Neurosci 36(5):275-284. 


\section{Figure legends}

Figure 1: Evolution of whole-brain morphometric parameters in the typical groups

For the pre-term, term, post-term and TEA groups, post-menstrual age (PMA) at MRI is summarized according to gestational age (GA) at birth (a). The dots code for the group belonging (triangles=pre-term, squares=term, circles=post-term, stars=TEA), and the sex is indicated by the color (pink=girl, blue=boy).

For the first three typical groups, hemispheric volume (b), cortical surface area (c), sulcation index (d) and SPANGY analyzed folding power (e) are presented as a function of PMA at MRI (the Gompertz models are outlined by the black curves in $d$ and e). The distribution of power analyzed with SPANGY across the bands B0 to B6, i.e. the power spectrum, is presented in (f).

\section{Figure 2: From MR images to SPANGY analyses}

The successive post-processing steps are outlined for typical babies at different postmenstrual ages (3 pre-term newborns at 27w, 30w and 34w, 1 term newborn at 39w, 3 infants at $47 \mathrm{w}, 52 \mathrm{w}$ and $60 \mathrm{w}$, all girls). T2w images (a), segmentations of the boundary between cortex and white matter (b) and 3D reconstructions of the inner cortical surface (c) are presented with real proportions across newborns. SPANGY segmentations (d) are projected on smoothed cortical meshes (zoomed proportions), showing B4 sulci elements (deep blue) even in the youngest newborns. Zooms on the region around the central sulcus (e) highlight the progressive appearance of parcels for B5 (light blue) and B6 (green).

\section{Figure 3: Evolution of SPANGY parameters in the three typical groups}

The characteristic wavelength (a), number of parcels (b), power (c) and power proportion (d) are presented for bands B4 (deep blue), B5 (light blue) and B6 (green) as a function of the newborns post-menstrual age. B4-B6 proportions are also represented in 3D with ellipsoids highlighting the 4 k-means clusters (e). Regarding the power law between cortical surface and brain size $\left(S=b V^{a}\right)$, different scaling exponents $a$ were observed in these 4 clusters (f). The dots code for the group (see Figure 1). For the numbers of parcels (b) and powers (c), the curves outline the Gompertz models, while in (d) they correspond to ratios between Gompertz models to highlight each band power proportions. 
Figure 4: Visual comparison of full-term newborns versus premature infants at TEA

As in Figure 1, T2w images (a), segmentations of the cortical boundary (b), 3D reconstructions of the inner cortical surface (c) and SPANGY segmentations (d) are outlined for 2 full-terms (39.4w and 38.5w PMA) and 2 preterms at TEA (41w, 40.5w PMA) (all boys). The zooms on the central sulcus (e) suggest simpler folding patterns in premature infants compared with full-term newborns despite equivalent hemispheric volumes (333 versus $334 \mathrm{~mL}, 371$ versus $379 \mathrm{~mL}$ ).

\section{Figure 5: Atypical cortical folding in premature infants at TEA}

Whole-brain parameters (a: hemispheric volume, cortical surface area, sulcation index) and B4-6 bands characteristics (b: number of parcels, c: power, d: power proportion) are presented as a function of PMA for premature infants at TEA (in black) compared with babies of the pre-term, term and post-term groups (in grey). Premature infants at TEA clearly deviate from the typical folding trajectory (Gompertz models from Figures 1 and 3 are outlined by grey curves), for the sulcation index and B4 power (see also Table 4).

\section{Table legends}

\section{Table 1: ANCOVA on the dependence to age and sex}

Analyses of variance were conducted over the pre-term, term and post-term groups jointly to evaluate whether each parameter depends on age and sex. For each co-variable and factor, the F value is outlined with the corresponding $\mathrm{p}$-value, and p-values were corrected for multiple comparisons with a FDR approach considering the 16 parameters of interest (i.e. considering each co-variable and factor independently). Statistically significant p-values suggesting linear dependence on age or sex are outlined in red (threshold $\mathrm{p}<0.05$ ).

\section{Table 2: Characteristic age points}

Gompertz modeling over the pre-term, term and post-term groups provided us with distinct age points (acceleration, inflection and deceleration points in weeks of PMA, with confidence intervals within brackets) for the sulcation index (SI), the analyzed folding power (AFP), the number of parcels for B5 and B6, and B4-6 power. 
Table 3: Characteristic wavelengths for bands B4-6

Minimal and maximal values of characteristic wavelengths over the pre-term, term and post-term groups are detailed for bands B4, B5 and B6, as well as the spatial resolution expected to be covered with SPANGY. For comparison, wavelengths over ICBM adult group are reminded (Germanaud, et al. 2012).

\section{Table 4: Deviations in folding development in premature infants at TEA}

For each parameter, we performed shuffle and split (S\&S) analysis to compare the group of premature infants at TEA with the typical trajectories of pre-term, term and postterm infants (significant $\mathrm{p}$-values are outlined in red for threshold $\mathrm{p}<0.05$, and with $*$ for $\mathrm{p}<0.005$ corresponding to Bonferroni correction for 10 comparisons). Results suggested deviations in the folding properties, particularly related to lower B4 power. Results from paired T tests, notably for power proportions, are provided in Supplementary Information \#2. Abbreviations: AFP analyzed folding power, SI sulcation index. 